\title{
Single-letter recognition accuracy benefits from advance cuing of location
}

\author{
A. H. C. VAN DER HEIJDEN, G. WOLTERS, J. C. GROEP, and R. HAGENAAR \\ University of Leiden, Leiden, The Netherlands
}

\begin{abstract}
With single-item visual displays, the facilitating effects of foreknowledge of item location have been demonstrated by using latency and accuracy as the dependent variables in detection tasks and by using latency as the dependent variable in recognition tasks. Evidence of such facilitating effects obtained by using accuracy as the dependent variable in recognition tasks is scarce and rather ambiguous. One of the difficulties in demonstrating the beneficial effects of location cuing in recognition accuracy may be the fact that in these tasks a performance baseline of no cuing is used that leaves only relatively little room for improvement. Therefore, in the present experiments, we varied the performance baseline by presenting letters on one of three imaginary circles at different distances from the fixation point, and determined, for each subject, a presentation time that resulted in an overall no-cue accuracy rate of .75 . In the experimental trials, three cue conditions were used: position cue (cuing 1 of 15 possible positions), ring cue (cuing 5 positions, all on one of the three circles), and no cue (cuing all 15 possible locations). In Experiment 1, the cue conditions were randomized. In Experiment 2, the cue conditions were blocked. Significant beneficial effects of foreknowledge of position were found in both experiments. Beneficial effects of ring cuing were found only in Experiment 2. In both experiments, the benefits of cuing are positively related to room for improvement. Partial correlations show that it is, indeed, room for improvement, and not the covarying factor of distance from fixation point, that determines cuing benefits. The theoretical implications of the beneficial effects of ring cuing under blocked presentation conditions are discussed.
\end{abstract}

In the present study, we used single-letter displays, that is, "empty" visual fields, to investigate central attentional effects on recognition accuracy in vision. In this context, attention can be operationally defined as the benefit in recognition or detection performance that occurs when subjects are cued in advance as to the location of a relevant stimulus (Prinzmetal, Presti, \& Posner, 1986). Central attentional effects are demonstrated if benefits are obtained in an experimental setup that excludes directed eye movements.

The information processing approach has provided clear evidence for central attentional effects with single-item displays in vision. In at least three types of task, clear benefits were obtained when subjects were given foreknowledge of where the single item was going to appear. Eriksen and Hoffman $(1973,1974)$ were the first to demonstrate faster reaction times in a letter-naming task, that is, in a suprathreshold recognition task, with latency as the dependent variable. Van der Heijden and Eerland (1973) and Bashinski and Bacharach (1980), among others, demonstrated improved accuracy in flash- and dotdetection tasks, that is, in threshold detection tasks, with accuracy as the dependent variable. Posner and his associates (see, e.g., Posner, Nissen, \& Ogden, 1978, and

The authors' mailing address is Department of Psychology, Unit of Experimental Psychology, University of Leiden, Hooigracht 15, 2312 KM Leiden, The Netherlands.
Posner, Snyder, \& Davidson, 1980; see also Posner, 1980) demonstrated improvements in reaction times to luminance increments, that is, in suprathreshold detection tasks, with latency as the dependent variable.

However, for one type of single-item task-the threshold recognition, or acuity, task using accuracy as the dependent variable-the evidence for central attentional involvement is scarce and rather ambiguous. Grindley and Townsend (1968, Experiment I) failed to find any positive effect on accuracy with foreknowledge of where a near-to-threshold acuity object (a T in one of four orientations) would appear (see Van der Heijden, Schreuder, \& Wolters, 1985, for a critical evaluation of this study). In a partially related study in which approximate foreknowledge of position was given by precuing one-third of the possible target positions, Egly and Homa (1984) found a positive effect with randomized cuing conditions (an average improvement of $4.1 \%$ ) but not with blocked cuing conditions $(0.3 \%)$. In a more elaborate replication of Grindley and Townsend's study with letters as the tobe-recognized items, Van der Heijden et al. (1985) found a small (3.2\%), but significant, improvement in recognition accuracy. In two subsequent experiments, however, in which various methods of backward masking were used to limit effective exposure duration, only very small and nonsignificant effects of foreknowledge of position were obtained (Fleur, Lapre, Van der Heijden, \& Wolters, 1986; Van Werkhoven, Wolters, \& Van der Heijden, 1986). 
For two reasons, we decided to again investigate central attentional effects, that is, effects of foreknowledge of position, in a single-item recognition task, using accuracy as the dependent variable. Our first reason for doing this was the theoretical importance of the early versus late selection issue in current theorizing in visual information processing (see, e.g., Van der Heijden, 1987). For example, Posner (1980) has suggested that foreknowledge of position might be important in simple (detection) tasks but not in complex (recognition) tasks, since in the latter case attention intervenes in a late stage where position information is no longer effective. Our second reason was that it was not clear why consistent and unambiguous effects of central selective attention had been demonstrated in single-item recognition tasks with latency as the dependent variable (see, e.g., Eriksen \& Hoffman, 1973, 1974; Posner, 1980; Posner et al., 1978; Tsal, 1983) but not with accuracy as the dependent variable.

With regard to the ambiguous results of location cuing on recognition accuracy, there is an important methodological point that easily goes unnoticed, namely room for improvement. The room for improvement is smaller in tasks that use accuracy as the dependent variable than in tasks that use latency. This is so because, with both dependent variables, performance in the foreknowledge, or cuing, condition has to be compared with baseline performance in a no-foreknowledge, or no-cuing, condition for benefits to be estimated. With accuracy as the dependent variable, an exposure duration is chosen in such a way that a baseline of performance without cuing, Pnc, results (a typical value of Pnc is .75). With foreknowledge of position, no improvement is possible in the proportion of Pnc on the trials that already result in correct responding without foreknowledge of position. So, with accuracy as the dependent variable, there is room for improvement only of a proportion $(1-\mathrm{Pnc})$ of the trials. With latency as the dependent variable, however, there is room for improvement in all trials. In summary, for the tasks we are discussing, latency seems a much more sensitive dependent variable (all trials can, in principle, contribute) than accuracy (only a porportion (1-Pnc) can contribute).

To see whether 1-Pnc, that is, room for improvement, is indeed the factor that makes it difficult for effects of central attention to show up, we reanalyzed the Van der Heijden et al. (1985), Van Werkhoven et al. (1986), and Fleur et al. (1986) data. In these experiments, effective exposure duration was manipulated in order to obtain, for each subject, a baseline performance, Pnc; of .75, or a room-for-improvement value $(1-\mathrm{Pnc})$ of .25 . That procedure, however, was never completely successful, inasmuch as some subjects in the main experiments showed somewhat higher and others somewhat lower baseline performances. Our reanalysis capitalized on these individual differences. We determined the linear functions relating the benefits (or costs) brought by foreknowledge of position per subject, $\Delta p$, to each subject's room for improvement $(1-\mathrm{Pnc})$. The results were quite surprising. In all experiments, a similar linear relationship was observed. The functions are

$$
\begin{aligned}
\Delta \mathrm{p}=.178(1-\mathrm{Pnc})-.016 & (r=.783) \\
\Delta \mathrm{p}=.259(1-\text { Pnc })-.067 & (r=.588) \\
\Delta \mathrm{p}=.321(1-\mathrm{Pnc})-.093 & (r=.677) \\
\Delta \mathrm{p}=.342(1-\mathrm{Pnc})-.056 & (r=.419)
\end{aligned}
$$

for the results reported in Van der Heijden et al. (1985), Fleur et al. (1986) Condition 1 and Condition 2, and Van Werkhoven et al. (1986), respectively (see also the data reported by Bashinski \& Bacharach, 1980, and Egly \& Homa, 1984, for similar relationships).

These results strongly suggest that it is indeed room for improvement that is the prime limiting factor in singleitem recognition tasks using accuracy as the dependent variable; subjects with below average baseline performance show larger benefits from advance position information, and subjects with above average baseline performance show smaller benefits from such information. In all cases, the benefits found for a subject equal a fraction of the proportion of trials that were not correct without foreknowledge of position (minus a constant proportion).

As stated, these results capitalized on small, unintended, between-subjects variations in baseline performance. To see whether the relationship really exists, it would be necessary to determine whether the same results would be obtained with larger within- and between-subject variations in baseline performance. We investigated this issue in the two experiments reported below. In our earlier experiments, single letters were presented at 1 of 5 locations on an imaginary circle around a fixation point. In the present experiments, 15 target positions were used. Baseline performance was varied by presenting the letters at 1 of 5 locations on one of three imaginary circles at different distances from the fixation point: ring 1 at $1^{\circ}$, ring 2 at $2^{\circ}$, and ring 3 at $3^{\circ}$. For each subject, an exposure duration was determined such that his/her overall baseline performance was about .75. Due to differential retinal acuity, baseline performance was much higher for ring 1 and much lower for ring 3. This also ensured that, especially for ring 3, there would be sufficient room for improvement for a reliable benefit to emerge (see, e.g., Egly \& Homa, 1984).

We investigated two further, subordinate, issues, both inspired by the results reported by Egly and Homa (1984), who also used three imaginary circles to position items.

Egly and Homa used two conditions, one with cued and uncued trials randomized and the other with the two cue types blocked. They obtained clear benefits only in the randomized condition. In the blocked condition, an interaction between practice and cue types may have prevented a main effect of foreknowledge of position to show up. This could be because Egly and Homa administered relatively few practice trials. We also used a blocked and a random condition, but in our experiments 
subjects received extensive practice prior to data collection to enable reliable effects.

Moreover, Egly and Homa presented foreknowledge only of the ring and not of the exact location of the impending target. So in their cued trials, the area that could contain the target was only partially reduced. Nevertheless, in their random condition, a clear benefit of this partial foreknowledge of location was found. In light of other attempts to demonstrate benefits in recognition tasks using accuracy as the dependent variable, this result is quite surprising. To see whether this effect was reliable, we also included a ring-cue condition. Thus, the resulting three cuing conditions were: (1) a position-cue condition, (2) a ring-cue condition, and (3) a no-cue condition.

\section{EXPERIMENT 1}

The prime purpose of this experiment was to investigate whether, in a recognition task using accuracy as the dependent variable, substantial benefits, due to foreknowledge of position, would be obtained if there was much room for improvement, 1-Pnc, or a low baseline performance level, Pnc.

Three cuing conditions were used: position cuing, ring cuing, and no cuing. To optimize conditions for a positive effect of cuing to show up, the three cuing conditions were completely randomized. Both Egly and Homa (1984), with ring cues, and Van der Heijden et al. (1985), with position cues, had demonstrated significant benefits in experimental conditions with cues completely randomized.

\section{Method}

Subjects. Nine students at the University of Leiden served as paid subjects. All had normal or corrected-to-normal vision.

Apparatus. The stimuli were presented on a fast-display screen (Vector General) equipped with fast-decaying P4-phosphor. Stimulus presentation and response registration were controlled by a PDP$11 / 34$ computer. The subjects initiated stimulus exposure by pressing a button in the table surface in front of them.

Stimuli and Experimental Conditions. Each stimulus consisted of one single capital letter chosen from a pool of eight letters, as used by Van der Heijden et al. (1985). The letters were E, F, H, $\mathrm{M}, \mathrm{O}, \mathrm{Q}, \mathrm{K}$, and $\mathrm{X}$.

A letter subtended maximal visual angles of $.28^{\circ}$ (width) and $.34^{\circ}$ (height) at a viewing distance of $92 \mathrm{~cm}$.

A letter was presented in one of five equally spaced positions on the circumference of one of three imaginary circles around a fixation point (a dim asterisk). The three imaginary circles had radii of $1^{\circ}, 2^{\circ}$, and $3^{\circ}$ of visual angle. This provided for 15 letter positions at three distances from the fixation point.

Low-intensity dots were used as position indicators. One, 5 , or 15 dots were presented, indicating, respectively, target position only (position-cue condition), all 5 positions on the imaginary circle containing the target position (ring-cue condition), or every position on every circle (no-cue condition). The radii of the imaginary circles were slightly larger for the position-indicating dots than for the letters.

Design. A within-subject design with repeated measures was employed. In each daily session, a subject received 360 trials - the factorial combination of 8 letters, 15 locations ( 5 positions on each of the 3 imaginary concentric circles), and 3 cue conditions.
Presentations were made in three successive blocks of 120 trials. The order in which the trials were administered was determined randomly for each subject and each session, the only constraint being that within a block each ring and each cue condition occur equally often.

Procedure. Each subject served on 4 consecutive days. The first day was used for practice only. During this practice session, the $75 \%$ correct recognition threshold was determined by varying the presentation times of letters in the no-cue condition (15 dots).

On each of the 3 experimental days, the subject was presented with three blocks of 120 trials. The presentation sequence per trial consisted of cue exposure for $25 \mathrm{msec}$, a subsequent delay of $75 \mathrm{msec}$, during which the screen was empty, and exposure of a letter in one of the 15 locations. The fixation point disappeared upon cue exposure and reappeared after letter exposure. The subject was required to respond by naming the letter. For each subject, the letterexposure duration determined on the first day was used. Durations ranged between 9 and $29 \mathrm{msec}$ with a mean of $15.3 \mathrm{msec}$.

Prior to each of the three blocks of experimental trials, practice trials were administered. A practice block consisted of 45 randomly ordered trials, with each cue condition and each letter position occurring equally often. In this practice block, exposure duration for each subject was reduced from 2 times that of his/her $75 \%$ recognition threshold for the first 15 stimuli, to $1 \frac{1 / 2}{2}$ times that duration for the second 15 stimuli, and to the $75 \%$ threshold time for the third 15 stimuli.

A short rest period followed each experimental block and preceded the next practice block. Except for the first day, no feedback was provided.

\section{Results and Discussion}

Correct-letter-identification scores were determined for each subject per experimental condition and per day. Each of these scores was based on $\mathbf{4 0}$ trials. An ANOVA over these data showed that the effect of days was not significant $[F(2,16)=1.8]$. Moreover, days did not interact with any of the experimental conditions. Therefore, it can be concluded that the extensive training procedure effectively eliminated practice effects in the experimental sessions.

Mean percentages of correct letter identification per subject and per experimental condition (collapsed over days) are given in Table 1 . Both experimental main effects, type of cue $[F(2,16)=62.2, p<.001]$ and distance of target letter from fixation point $[F(2,16)=148.1$, $p<.001]$, are highly significant. Mean percentages of correct identification in the no-cue, ring-cue, and positioncue conditions are $73.3,72.9$, and 80.7 , respectively. An a posteriori comparison of these means (Newman-Keuls) showed that only the differences of position cue versus no cue and those of position cue versus ring cue are significant (both $p<.01$ ), indicating that only positioncuing shows a facilitatory effect on letter-identification performance. So, contrary to Egly and Homa (1984), no beneficial effect of ring-cuing is found.

Mean percentages of correct identification for the shortest (ring 1), intermediate (ring 2), and longest (ring 3) distances of targets from fixation point are 89.2, 77.1, and 60.6, respectively. A Newman-Keuls test showed that all of these means differ significantly at the .01 level, indicating a monotonic decrease in performance with an increase in distance of target letters from the fixation point. 
Table 1

Mean Percentage of Correct Letter Identification Per Subject as a Function of Cuing Conditions and Distance from Fixation Point (Experiment 1: Cue Conditions Random)

\begin{tabular}{|c|c|c|c|c|c|c|c|c|c|}
\hline \multirow[b]{2}{*}{ Subject } & \multicolumn{3}{|c|}{ No-Cue Condition } & \multicolumn{3}{|c|}{ Ring-Cue Condition } & \multicolumn{3}{|c|}{ Position-Cue Condition } \\
\hline & Ring 1 & Ring 2 & Ring 3 & Ring 1 & Ring 2 & Ring 3 & Ring 1 & Ring 2 & Ring 3 \\
\hline 1 & 91.7 & 72.5 & 52.5 & 84.2 & 82.5 & 50.8 & 89.2 & 75.8 & 67.5 \\
\hline 2 & 92.5 & 83.3 & 60.0 & 89.2 & 85.8 & 58.3 & 90.0 & 85.8 & 81.7 \\
\hline 3 & 82.5 & 70.8 & 49.2 & 86.7 & 79.2 & 45.0 & 93.3 & 75.8 & 67.5 \\
\hline 4 & 90.8 & 76.7 & 58.3 & 89.2 & 75.8 & 50.0 & 90.8 & 70.8 & 77.5 \\
\hline 5 & 87.5 & 71.7 & 40.8 & 78.3 & 75.8 & 48.3 & 88.3 & 84.2 & 60.0 \\
\hline 6 & 85.0 & 75.8 & 59.2 & 85.0 & 70.0 & 53.3 & 88.3 & 75.8 & 76.7 \\
\hline 7 & 91.7 & 73.3 & 47.5 & 93.3 & 65.8 & 44.2 & 84.2 & 70.8 & 73.3 \\
\hline 8 & 92.5 & 77.5 & 65.8 & 95.8 & 76.7 & 67.5 & 93.3 & 90.8 & 77.5 \\
\hline 9 & 94.2 & 76.7 & 59.2 & 90.0 & 82.5 & 65.0 & 91.7 & 80.0 & 79.2 \\
\hline Mean & 89.8 & 75.4 & 54.7 & 88.0 & 77.1 & 53.6 & 89.9 & 78.9 & 73.4 \\
\hline
\end{tabular}

Also, the interaction between cuing conditions and distance of target to fixation point is significant $[F(4,32)=$ $15.5, p<.001]$. As can be inferred from Figure 1, this interaction is due to increasing beneficial effects of position-cuing over rings. The benefits of position-cuing compared to no cuing are $.1 \%, 3.5 \%$, and $18.7 \%$ for rings 1,2 , and 3 , respectively.

The results clearly show that position-cuing enhances subsequent identification performance, and it appears that this enhancement increases as room for improvement increases. This is substantiated by the linear function relating benefits from position-cuing, $\Delta p$, and room for improvement, 1-Pnc. This function is

$$
\Delta \mathrm{p}=.518(1-\mathrm{Pnc})-.064 \quad(r=.843) .
$$

Note that the form of this function is similar to that of the functions obtained in our earlier experiments (see the introduction). However, in the present experiment, room for improvement covaries with distance of targets from fixation point. To differentiate between the effect of room for improvement (1-Pnc) and distance of target from fixation point $(R)$, correlations and partial correlations were

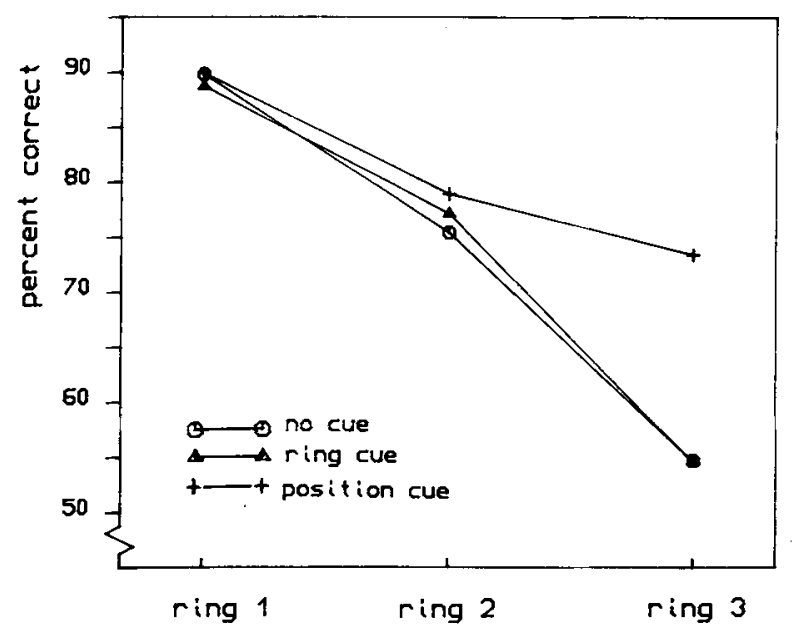

Figure 1. Mean percentages of correct letter identification (Experiment 1, cue conditions random). determined between performance enhancement due to the presence of a position cue $(\Delta \mathrm{p})$ and $1-$ Pnc and between $\Delta \mathrm{p}$ and $R$. These correlations are:

$$
\begin{array}{ll}
r(\Delta \mathrm{p})(1-\mathrm{Pnc})=.843 & r(\Delta \mathrm{p})(1-\mathrm{Pnc}) \cdot(R)=.433 \\
r(\Delta \mathrm{p})(R)=.804 & r(\Delta \mathrm{p})(R) \cdot(1-\mathrm{Pnc})=.076
\end{array}
$$

It is clear that performance enhancement due to the presence of a position cue is related more to $1-$ Pnc than to $R$. Although partialing out $R$ decreases the relationship between $\Delta \mathrm{p}$ and 1-Pnc, nullifying 1-Pnc results in a much larger decrease in, and actually a complete disappearance of, the relationship between $\Delta \mathrm{p}$ and $R$. Additional evidence that position-cuing benefits are determined by room for improvement (or baseline performance) and not by ring is the fact that the relationship between $\Delta p$ and 1-Pnc found in this experiment (see Function 1) is similar to the relationships we found in our earlier experiments, in which only one distance from the fixation point (one ring) was used.

Taken together, foreknowledge of location by cuing the position of an impending target letter is beneficial for letter identification in conditions in which the possibility of eye movements is excluded. This beneficial cuing effect is a function of the available room for improvement. Large benefits are found with a large room for improvement.

\section{EXPERIMENT 2}

Unlike Egly and Homa (1984), we found, in Experiment 1 , no benefit with the ring cue. One reason for this null result may be that subjects cannot simultaneously maintain three different optimal processing strategiesone for no-cue trials, one:for ring-cue trials, and one for position-cue trials. Egly and Homa obtained benefits with ring-cuing in conditions in which only two strategies had to be maintained-one for no cuing and one for ring-cuing. To investigate this issue, an experiment with blocked cuing conditions seemed appropriate. This would make it possible for the subjects to use one unique and optimal processing strategy in each cuing condition. So the prime purpose of Experiment 2 was to determined whether cu- 
Table 2

Mean Percentage of Correct Letter Identification Per Subject as a Function of Cuing Conditions and Distance from Fixation Point (Experiment 2: Cue Conditions Blocked)

\begin{tabular}{|c|c|c|c|c|c|c|c|c|c|}
\hline \multirow[b]{2}{*}{ Subject } & \multicolumn{3}{|c|}{ No-Cue Condition } & \multicolumn{3}{|c|}{ Ring-Cue Condition } & \multicolumn{3}{|c|}{ Position-Cue Condition } \\
\hline & Ring 1 & Ring 2 & Ring 3 & Ring 1 & Ring 2 & Ring 3 & Ring 1 & Ring 2 & Ring 3 \\
\hline 1 & 93.3 & 83.3 & 68.3 & 93.3 & 87.5 & 70.8 & 91.7 & 78.3 & 66.7 \\
\hline 2 & 94.2 & 75.8 & 56.7 & 90.8 & 79.2 & 58.3 & 94.2 & 81.7 & 65.0 \\
\hline 3 & 90.0 & 79.2 & 65.8 & 92.5 & 83.3 & 67.5 & 95.0 & 82.5 & 62.5 \\
\hline 4 & 90.0 & 80.8 & 69.2 & 92.5 & 86.7 & 69.2 & 92.5 & 87.5 & 73.3 \\
\hline 5 & 96.7 & 82.5 & 66.7 & 95.0 & 85.8 & 71.7 & 96.7 & 81.7 & 67.5 \\
\hline 6 & 88.3 & 81.7 & 55.0 & 87.5 & 77.5 & 61.7 & 92.5 & 83.3 & 65.0 \\
\hline 7 & 90.8 & 80.8 & 72.5 & 94.2 & 85.8 & 77.5 & 94.2 & 80.0 & 76.7 \\
\hline 8 & 85.0 & 70.0 & 47.5 & 85.0 & 70.8 & 50.0 & 79.2 & 76.7 & 60.0 \\
\hline 9 & 85.0 & 77.5 & 45.0 & 90.8 & 79.2 & 55.8 & 94.2 & 80.8 & 65.0 \\
\hline Mean & 90.4 & 79.1 & 60.7 & 91.3 & 81.8 & 64.7 & 92.2 & 81.4 & 66.9 \\
\hline
\end{tabular}

ing effects would show up when cuing conditions were blocked instead of randomized and when extensive practice was given to prevent an interaction effect between practice and cuing conditions.

\section{Method}

Subjects. Nine students at the University of Leiden, who had not participated in the previous experiment, served as paid subjects. All had normal or corrected-to-normal vision.

Apparatus, Stimuli, and Experimental Conditions. Apparatus, stimuli, and cue displays were identical to those used in Experiment 1 .

Design. The design of Experiment 2 was the same as that of Experiment 1 , except that presentation was blocked for cue conditions. In each daily experimental session, each subject was tested in three blocks of 120 trials, one for each cue condition. Within a block, the order of distances of targets to the fixation point was randomized. Per day, order of blocks was balanced over subjects; per subject, order was balanced over days.

Procedure. The procedure was similar to that in Experiment 1. The $\mathbf{4 5}$ practice trials preceding the experimental trials were correspondingly blocked for cue condition.

\section{Results and Discussion}

The procedure for determining correct identification scores was the same as in Experiment 1.

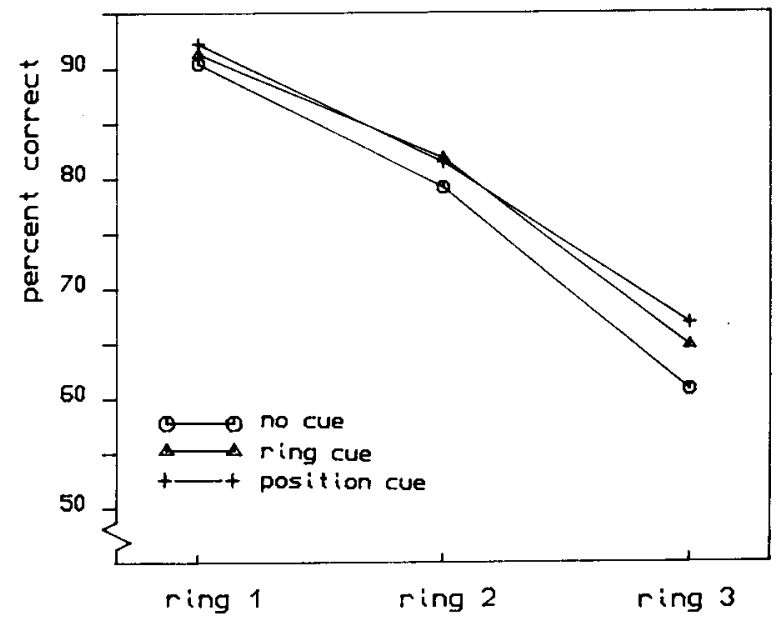

Figure 2. Mean percentages of correct letter identification (Experiment 2 , cue conditions blocked).
The extensive periods of practice before experimental trials were administered again proved effective in eliminating practice effects over experimental sessions. No significant effect of days and no significant interaction effects between days and experimental variables were found. Mean percentages of correct letter identifications in the experimental conditions per subject are given in Table 2 .

Both main effects, type of cue $[F(2,16)=5.5, p<$ .05] and distance of target letter from fixation point $[F(2,16)=169.7, p<.001]$, are significant. Mean percentages of correct identification of letters in the no-cue, ring-cue, and position-cue conditions are $76.7,79.2$, and 80.2 , respectively. Means of the ring-cue and positioncue conditions do not differ, but both differ significantly from the no-cue condition (Newman-Keuls test, $p<.05$ ). So, in contrast to Experiment 1, ring cues do result in enhanced identification performance when cuing conditions are blocked.

Mean percentages of correct identification for the shortest, intermediate, and longest distances of targets from fixation point are $91.3,80.8$., and 64.1 , respectively. All means differ significantly (Newman-Keuls test, $p<.01$ ), showing again a monotonic decrease in performance with increasing distances to the fixation point.

Although the interaction between cuing conditions and distance is not significant $[F(4,32)=1.8]$, inspection of Figure 2 seems to suggest that beneficial effects of both position-cuing and ring-cuing increase over rings, that is, they increase as room for improvement increases. The linear function relating benefits from position-cuing, $\Delta p$, and room for improvement, 1-Pnc, again is similar to the functions reported earlier. For the data in this experiment, the function is

$$
\Delta \mathrm{p}=.243(1-\mathrm{Pnc})-.023 \quad(r=.610) .
$$

To differentiate between the effects of room for improvement and distance of target from fixation point, correlations and partial correlations were again determined between performance enhancement due to position-cuing $(\Delta \mathrm{p})$ and $1-\mathrm{Pnc}$ and between $\Delta \mathrm{p}$ and $R$ :

$$
\begin{array}{ll}
r(\Delta \mathrm{p})(1-\mathrm{Pnc})=.610 & r(\Delta \mathrm{p})(1-\mathrm{Pnc}) \cdot(R)=.738 \\
r(\Delta \mathrm{p})(R)=.317 & r(\Delta \mathrm{p})(R) \cdot(1-\mathrm{Pnc})=-.589
\end{array}
$$


Nullifying the effect of $R$ enhances the relationship between $\Delta p$ and $1-$ Pnc. On the other hand, partialing out 1 -Pnc decreases, and in fact reverses, the relationship between $\Delta \mathrm{p}$ and $R$. This negative relationship actually weakens the relationship between $\Delta p$ and 1 -Pnc. Again, room for improvement, and not distance, is responsible for the beneficial effects of foreknowledge of position found in this experiment. If ring has any effect at all, it decreases the beneficial effect of position-cuing.

For the ring-cue condition, the linear function relating benefits, $\Delta \mathbf{r}$, and room for improvement is

$$
\Delta \mathrm{r}=.112(1-\mathrm{Pnc})-.001 \quad(r=.490) .
$$

As before, to disentangle the covariation between room for improvement and distance of target from fixation point, correlations and partial correlations were determined:

$$
\begin{array}{ll}
r(\Delta \mathrm{r})(1-\mathrm{Pnc})=.490 & r(\Delta \mathrm{r})(1-\mathrm{Pnc}) \cdot(R)=.323 \\
r(\Delta \mathrm{r})(R)=.397 & r(\Delta \mathrm{r})(R) \cdot(1-\mathrm{Pnc})=-.085
\end{array}
$$

Also, for the ring-cue effect, it can be concluded that performance enhancement is related to room for improvement and not to distance of target from the fixation point, since, by nullifying the effect of $1-$ Pnc, the relationship between $\Delta \mathrm{r}$ and $R$ disappears.

Taken together, under blocked cuing conditions, not only position cues, but also ring cues, enhance identification performance. Both cuing effects are a function of the available room for improvement.

\section{GENERAL DISCUSSION}

One of our reasons for reinvestigating the effects of precise foreknowledge of position in a single-item recognition task with accuracy as the dependent variable was that reliable effects had been demonstrated with latency as the dependent variable but not with accuracy as the dependent variable. We suggested that this difference in results might have been caused by differences in room for improvement in the two types of tasks.

The results obtained in both experiments clearly support this suggestion. The correlations show that benefits increase with room for improvement. The partial correlations further show that it is, indeed, room for improvement, and not distance from the fixation point, that is the factor responsible; distance from the fixation point has either no effect or a detrimental effect. Large and reliable benefits are obtained if there is large room for improvement. Therefore, our results show that there is nothing special about a single-item recognition-accuracy task relative to a latency task.

Another reason for again investigating the effects of foreknowledge of position in a single-item recognition task with accuracy as the dependent variable is the potential theoretical importance of this task in shedding light on the early versus late selection issue (see, e.g., Van der Heijden, 1987). Posner (1980; Posner et al., 1980) has suggested that, with this type of task, early information, that is, foreknowledge of position, is not very useful and that "this is due to the necessity of the subject's switching attention from the spatial location indicated by the cue to the internal lookup processes that identify ... or determine ... the discriminative responses" (Posner et al., 1980 , p. 168). So, in their view, attention can effectively intervene only late in the present type of task. It will be clear that our results do not support this view. Significant and substantial benefits, up to $18.7 \%$, were demonstrated. In this way, our results strongly suggest that early location and physical information is very useful in singleitem recognition tasks with accuracy as the dependent variable. This is consistent with the proposals of early selection models. However, this result is also consistent with Van der Heijden's (see, e.g., 1981, 1984, 1987; Van der Heijden, Hagenaar, \& Bloem, 1984) postcategorical filtering model, which postulates that identity information has to be addressed via early visual information.

A final reason for conducting the research here reported was the finding, reported by Egly and Homa (1984), of benefits with partial foreknowledge of position, that is, with a ring cue. To see whether this effect was reliable, we included ring-cue conditions in both experiments. In our first experiment, with random-cuing conditions, no positive effect of ring-cuing was found. In our second experiment, with cuing conditions blocked, however, ringcuing produced significant benefits. The results obtained in the ring-cue condition were essentially the same as those in the position-cue condition.

We have already hinted at an explanation of why an effect of ring-cuing might have shown up in the blockedpresentation condition but not in the random-presentation condition. In the random-presentation condition, subjects have to maintain three selection strategies. This task may be too difficult for subjects to perform. One means of reducing the task difficulty is to consider both the 5-dot ring-cue condition and the 15-dot no-cue condition as nocue conditions, thereby reducing the task to a twocondition, cue versus no-cue, task. (In this context, it is important to note that Egly and Homa, 1984, found benefits with ring-cuing in a two-condition ring-cue versus no-cue task.) In the blocked presentation condition, subjects can maintain one optimal selection strategy per block. In this case, a reliable effect of ring-cuing can be demonstrated.

According to Egly and Homa (1984), their data suggest that attention can be simultaneously allocated to multiple regions of the visual field or that broad visual fields with different shapes and sizes can be simultaneously sensitized. Posner et al. (1980), on the other hand, concluded that attention cannot be allocated to multiple regions simultaneously. They compared attention to a spotlight that enhances detection of events only within its beam. Eriksen and Hoffman $(1972,1973)$ obtained evidence, in a number of experiments, that this spotlight is of a fixed minimal size. It is important to realize that the positive effects of ring-cuing repotted by Egly and Homa (1984) and found 
in our Experiment 2 need not necessarily be explained in terms of sensitization of multiple regions or broad visual fields. It is also possible to explain these effects in terms of a spotlight with a fixed size. Such an explanation runs as follows.

Before attention can move to the position of the (impending) target, the location of the target has to be known. Assume that, in the system responsible for programming this movement, polar coordinates, not euclidean coordinates, are used. With ring-cuing blocked, one of the two parameters needed, the distance $\delta$, is provided in advance. The second parameter, the angle of the movement $\phi$, can be programmed only after presentation of the letter. In the no-cuing condition, none of the parameters is available in advance. Given some further assumptions about the time needed for programming and/or the capacity of the programmer, any cuing effect, including an advantage for the ring-cuing condition over the no-cuing condition, may be obtained. This argument is not intended to show that multiple regions cannot be sensitized simultaneously or that attentional movements are programmed in polar coordinates. The important point is that ring-cuing does not provide decisive information with regard to these issues and that further experimentation is needed.

\section{REFERENCES}

Bashinski, H. S., \& BaCharach, V. R. (1980). Enhancement of perceptual sensitivity as the result of selectively attending to spatial locations. Perception \& Psychophysics, 28, 241-248.

EGLY, R., \& HomA, D. (1984). Sensitization of the visual field. Journal of Experimental Psychology: Human Perception \& Performance, 10, 778-793.

ERIKSEN, C. W., \& HoFFman, J. E. (1972). Temporal and spatial characteristics of selective encoding from visual displays. Perception \& Psychophysics, 12, 201-204.

ERIKSEN, C. W., Hoffman, J. E. (1973). The extent of processing of noise elements during selective encoding from visual displays. Perception \& Psychophysics, 14, 155-160.

ERIKSEN, C. W., \& HofFMAN, J. E. (1974). Selective attention: Noise suppression or signal enhancement? Bulletin of the Psychonomic Society, 4, 587-589.
Fleur, E., Lapré, L., Van der Heuden, A. H. C., \& Wolters, G. (1986). The effect of different numbers of cues and different masking patterns on letter recognition (Internal report, EP-86-02). Leiden, The Netherlands: University of Leiden.

GrINDLEY, G. C., \& TownSEND, V. (1968). Voluntary attention in peripheral vision and its effects on acuity and differential thresholds. Quarterly Journal of Experimental Psychology, 20, 11-19.

PosNer, M. I. (1980). Orienting of attention. The VIIth Sir Frederic Bartlett Lecture. Quarterly Journal of Experimental Psychology, 32, 3-25.

Posner, M. I., Nissen, M. J., \& Ogden, W. C. (1978). Attended and unattended processing modes: The role of set for spatial location. In H. L. Pick \& I. J. Saltzman (Eds.), Modes of perceiving and processing information. Hillsdale, NJ: Erlbaum.

Posner, M. I., SNyder, C. R. R., \& Davidson, B. J. (1980). Attention and the detection of signals. Journal of Experimental Psychology: General, 109, 160-174.

Prinzmetal, W., Presti, D. E. \& Posner, M. I. (1986). Does attention affect visual feature integration? Journal of Experimental Psychology: Human Perception \& Performance, 12, 361-369.

TSAL, Y. (1983). Movements of attention across the visual field. Journal of Experimental Psychology: Human Perception \& Performance, 9, 523-530.

VAN DER HEIJDEN, A. H. C. (1981). Short-term visual information forgetting. London: Routledge \& Kegan Paul.

VAN DER HeIJDEN, A. H. C., (1984). Postcategorical filtering in a barprobe task. Memory \& Cognition, 12, 446-457.

VAN DER HeIJDEN, A. H. C. (1987). Central selection in vision. In H. Heuer \& A. F. Sanders (Eds.), Perspectives on perception and action. Hillsdale, NJ: Erlbaum.

VAN der Heijden, A. H. C., \& Eerland, E. (1973). The effects of cueing in a visual signal detection task. Quarterly Journal of Experimental Psychology, 25, 496-503.

VAN der Heisden, A. H. C., HagenaAr, R., \& Bloem, W. (1984). Two stages in postcategorical filtering and selection. Memory \& Cognition, 12, 458-469.

VAN Der Heuden, A. H. C., Schreuder, R., \& Wolters, G. (1985). Enhancing single-item recognition accuracy by cueing spatial locations in vision. Quarterly Journal of Experimental Psychology, 37a, 427-434.

Van Werkhoven, S., Wolters, G., \& Van der Heiden, A. H. C. (1986). The influence of masking on recognition of cued letters (Internal report, EP-86-01). Leiden, The Netherlands: University of Leiden.

(Manuscript received January 12, 1987; revision accepted for publication May 18, 1987.) 